Journal for ImmunoTherapy of Cancer

\section{patients with advanced mismatch repair-deficient and repair-proficient endometrial cancer. A nonrandomized phase 2 clinical trial}

Yoland Antill (D) ,1,2 Peey-Sei Kok, ${ }^{3}$ Kristy Robledo, ${ }^{3}$ Sonia Yip, ${ }^{3}$ Michelle Cummins, ${ }^{3}$ Deborah Smith, ${ }^{4}$ Amanda Spurdle, ${ }^{5}$ Elizabeth Barnes, ${ }^{3}$ Yeh Chen Lee, ${ }^{3,6,7}$ Michael Friedlander, ${ }^{6}$ Sally Baron-Hay, ${ }^{8}$ Catherine Shannon, ${ }^{9}$ Jermaine Coward, ${ }^{10,11}$ Philip Beale, ${ }^{7}$ Geraldine Goss, ${ }^{12}$ Tarek Meniawy, ${ }^{13}$ Janine Lombard, ${ }^{14}$ John Andrews, ${ }^{3}$ Martin R Stockler, ${ }^{3}$ Linda Mileshkin, ${ }^{15}$ on behalf of the Australia New Zealand Gynaecological Oncology Group (ANZGOG)

To cite: Antill Y, Kok P-S, Robledo K, et al. Clinical activity of durvalumab for patients with advanced mismatch repair-deficient and repairproficient endometrial cancer. A nonrandomized phase 2 clinical trial. Journal for ImmunoTherapy of Cancer 2021;9:e002255. doi:10.1136/jitc-2020-002255

- Additional supplemental material is published online only. To view, please visit the journal online (http://dx.doi.org/10. 1136/jitc-2020-002255).

Some results of this study were presented at the American Society of Clinical Oncology Meeting 2019 as an oral presentation and at the European Society for Medical Oncology Asia 2019 meeting as a mini-oral presentation.

Accepted 31 March 2021

Check for updates

(C) Author(s) (or their employer(s)) 2021. Re-use permitted under CC BY-NC. No commercial re-use. See rights and permissions. Published by BMJ.

For numbered affiliations see end of article.

Correspondence to Associate Professor Yoland Antill; Yoland.Antill@monash.edu

\section{ABSTRACT}

Background In this study, we assessed the activity of durvalumab, an antibody to programmed death ligand-1, in two cohorts of women with advanced endometrial cancers (AEC) - mismatch repair proficient (pMMR) and mismatch repair deficient (dMMR).

Methods A multicenter phase two study was performed in women with AEC with pMMR tumor progressing after one to three lines of chemotherapy and women with AEC with dMMR tumor progressing after zero to three lines of chemotherapy. Mismatch repair status was based on immunohistochemistry expression. All women received durvalumab $1500 \mathrm{mg}$ given every 4 weeks until progression or unacceptable toxicity. The primary endpoint was objective tumor response by RECIST V.1.1 modified for immune-based therapeutics.

Results Seventy-one women were recruited: 35 dMMR and 36 pMMR. Median follow-up was 19 vs 21 months in dMMR versus pMMR, respectively. Median age was 67 years. Histology in dMMR versus pMMR included endometrioid ( $94 \%$ vs $57 \%$ ) and serous ( $0 \%$ vs $31 \%$ ) response rate (OTRR) in the dMMR cohort was 47\% (17/36, 95\% Cl 32 to 63), including 6 complete responses and 11 partial responses (PRs)) vs $3 \%$ in the pMMR cohort (1/35, 95\% $\mathrm{Cl} 1$ to $15, \mathrm{PR})$. In the dMMR cohort, durvalumab was the first-line therapy in 58\% (OTRR $57 \%$ ) and the second-line therapy in 39\% (OTRR 38\%). Median progression-free survival was 8.3 months in the dMMR cohort vs 1.8 months in the pMMR cohort. The 12 -month overall survival (OS) rate was $71 \%$ in dMMR vs $51 \%$ in pMMR, with median OS not reached for dMMR vs 12 months for pMMR. Immune-related adverse events occurred in 14 women, mostly grades 1-2.

Conclusion Durvalumab monotherapy showed promising activity and acceptable safety in AEC with dMMR regardless of prior lines of chemotherapy, but activity was limited in AEC with pMMR. and was high grade in $26 \%$ vs $74 \%$. The objective tumor
Trial registration numbers ANZG0G1601, ACTRN12617000106336, and NCT03015129.

\section{BACKGROUND}

The incidence of endometrial cancer (EC) has increased over recent decades, likely reflecting rising obesity rates worldwide. Historically, the descriptive terms type I and type II EC were used to distinguish cancers with endometrioid histology (type I) with a good prognosis, versus histological subtypes with a poorer prognosis, such as serous papillary and clear cell (type II). ${ }^{12}$ More recently, The Cancer Genome Atlas, using a combination of genomic, proteomic and epigenomic evaluations of primary EC, has suggested four distinct molecular subtypes: polymerase- $\varepsilon$ (POLE)-hypermutated, microsatellite instability (MSI), copy number-low/p53 wild type, and copy number-high/p53-mutated, which reflect tumor biology and prognosis more accurately than histological subtype and grade. ${ }^{3}$ Mismatch repair deficiency (dMMR) in the DNA repair pathway is present in $15 \%-30 \%$ of all ECs and perhaps a higher proportion of advanced or recurrent EC. ${ }^{4}$ Cancers with dMMR typically have a microsatellite instability-high (MSI-H) phenotype, due to high mutational frequency. While the majority of dMMR tumors are related to acquired $M L H 1$ hypermethylation, $13 \%-25 \%$ have been reported to relate to inherited germline mutations, with the remainder likely due to somatic mutations of one of the four mismatch repair genes (MLH1, MSH2, 
MSH6 or PMS2) or EPCAM (causing a downstream silencing of MSH2) ${ }^{5-10}$ These tumors also have a higher predicted neoantigen load, which generate strong CD3+ and CD8+ T-cell responses together predictive of immunotherapy sensitivity. ${ }^{11}$ There is increasing evidence that dMMR tumors are associated with chemotherapy resistance. ${ }^{12}{ }^{13}$ Compared with mismatch repair-proficient (pMMR) tumor, dMMR endometrial tumors may have a worse prognosis in the advanced setting. ${ }^{4}$

The interplay between tumor cells and the immune system, with evasion of normal immunological recognition and control, appears to be a keystone of tumorigenesis and metastasis. Interaction between ECs and the tumor microenvironment results in immune response modulation. Programmed death ligand-1 (PD-L1) is part of the B7-CD28 family and is a ligand for the programmed death receptor 1 (PD-1). Expression of PD-1 and PD-L1 vary among EC histological subtypes, molecular subclass and disease stage. ${ }^{1415}$ PD-1 activation and interaction with PD-L1/L2 inhibit and inactivate tumor infiltrating CD4 and CD8 T cells within the tumor microenvironment, protecting tumors from immune-regulatory control and destruction. $^{16}$

Treatment options are limited for women with advanced or recurrent EC, and outcomes are generally poor. Beyond progression after combined platinum and taxane-based therapy, observed response rates to singleagent cytotoxics are approximately $20 \%$ or lower, with significant treatment-related toxicities. ${ }^{17-20}$ Pembrolizumab (a PD-1 monoclonal antibody) was granted accelerated approval as the first tissue/site-agnostic approval in patients with MSI-H or dMMR cancers based on findings from five single-arm studies that reported durable responses. ${ }^{21-23}$ In pMMR or microsatelite stable cancers, PD-1 expression was associated with a modest response rate to PD-1 inhibition. ${ }^{23}$

Durvalumab (MEDI-4736) is a human IgG1 kappa monoclonal antibody directed against PD-L1. The PHAEDRA study (PHAse 2 trial of DuRvalumab in Advanced Endometrial Cancer) aimed to determine the activity of durvalumab in women with advanced endometrial cancer (AEC) that was either AMMR or pMMR.

\section{PATIENTS AND METHODS}

This phase II, multicenter, non-randomized, noncomparative study assessed the activity of durvalumab monotherapy in two cohorts of women with AEC. The two cohorts, based on immunohistochemical (IHC) expression of the four mismatch mutation repair (MMR) proteins (MLH1, MSH2, MSH6 and PMS2) were classified as either pMMR (expression of all four proteins) or dMMR (loss of at least one of the four proteins) (see Tumor Assessment). The key eligibility criteria were confirmed advanced or recurrent adenocarcinoma of the endometrium with target lesions according to RECIST V.1.1, ${ }^{24}$ not amenable to curative surgical resection, age $\geq 18$ years, Eastern Cooperative Oncology Group performance status of $0-2$, progressing after one to three lines of prior chemotherapy if pMMR or zero to three lines of prior chemotherapy if dMMR. The main exclusion criteria were carcinosarcoma histology, brain metastasis, history of another malignancy within the last 3 years and any contraindication to, or past treatment with, an immune checkpoint inhibitor.

\section{Tumor assessment}

All women provided written informed consent. Women were enrolled based on MMR status assessed at their recruiting site, using either tissue from a repeat biopsy prior to enrollment or archival tissue if a repeat biopsy was deemed unsafe for the patient. Assessment for MMR protein expression was completed according to local site protocols and varied between sites. The results reported here are based on a central review of MMR status using a single representative slide from tumor blocks used in cohort allocation.

\section{Treatment and assessments}

All women received durvalumab $1500 \mathrm{mg}$, given intravenously every 4 weeks until progression of disease, prohibitive toxicity, or withdrawal from the study. Radiological assessments for tumor response were performed every 8 weeks to week 24, then every 12 weeks until progression. Adverse events (AEs) were recorded according to Common Terminology Criteria for Adverse Events V.4.0 until 90 days after the last dose of durvalumab. Healthrelated quality of life (HRQL) was assessed with the European Organisation for Research and Treatment of Cancer- Quality of Life Questionnaire- C30 (EORTC QLQ-C30) at baseline, and then every 4 weeks until progression.

\section{Study endpoints}

The primary endpoint was objective tumor response (OTR) according to RECIST V.1.1 modified for immunebased therapeutics (iRECIST). ${ }^{25}$ Secondary endpoints included OTR according to standard RECIST 1.1, disease control at weeks 16 and 24, progression-free survival (PFS), overall survival (OS), duration of response and disease control, AEs and HRQL. Tertiary correlative studies to assess biomarkers (including PD-L1 expression) for associations with clinical outcomes, including OTR, duration of response and disease control, are ongoing and will be reported elsewhere.

\section{Statistical analysis}

Using a Simon's two-stage minimax design, 70 women in two separate cohorts of 35 with dMMR tumors and 35 with pMMR tumors, we allowed an OTR in each cohort of $5 \%$ or lower to be ruled out if the true rate is $20 \%$, with type 1 and type 2 error rates of $10 \%$, and an allowance of $10 \%$ for ineligibility and missing data. Accrual to a cohort was to be closed if no OTR were observed in the first 18 evaluable women. Durvalumab would be considered worthy of further research if four or more OTRs were observed in 
the first 32 evaluable women in a cohort (observed objective tumor response rate (OTRR) $12.5 \%$ or more).

Analyses for outcomes were by intention to treat including all women registered in each cohort. OTRR is defined as the proportion of women with an OTR (complete response (CR) or partial response (PR)) divided by the total number of women in that cohort. A response must be confirmed with consecutive scans at least 4 weeks apart (typically 8 weeks apart as per the schedule of assessments). Disease control included CR, PR or stable disease (SD) at 16 and 24 weeks. PFS was measured from registration until the date of the first documented progression or death from any cause and was summarized using the Kaplan-Meier method. Women were censored at the date they were last known to be progression-free. OS was from registration until death or the last date known to be alive. An improvement in a domain of HRQL assessed with the EORTC QLQ-C30 was defined as an improvement of $\geq 10$ points from baseline, on a scale from 0 to 100 , maintained for at least two subsequent visits.

\section{RESULTS \\ Subjects}

A total of 74 women were enrolled from 10 Australian centers between February 2017 and September 2018: 36 in the dMMR cohort (including 1 who was reassigned from pMMR to dMMR after central pathology review) and 35 in the pMMR cohort, where 71 were eligible and included for analysis (figure 1). Baseline characteristics are outlined in table 1 . The median ages were 66 years (range 36-76) and 68 years (range 37-81) in the dMMR and pMMR cohorts, respectively. The main histological subtype for dMMR was endometrioid in 34/36 (94\%), whereas for pMMR, the histology was endometrioid in 20/35 (58\%) and serous in $11 / 35$ (31\%). One subject in the pMMR group was subsequently found to have carcinosarcoma and therefore was ineligible but was included in all analyses. The majority of dMMR tumors were associated with loss of MLH1 and PMS2 $(78 \%)$. Previous platinum-based chemotherapy was

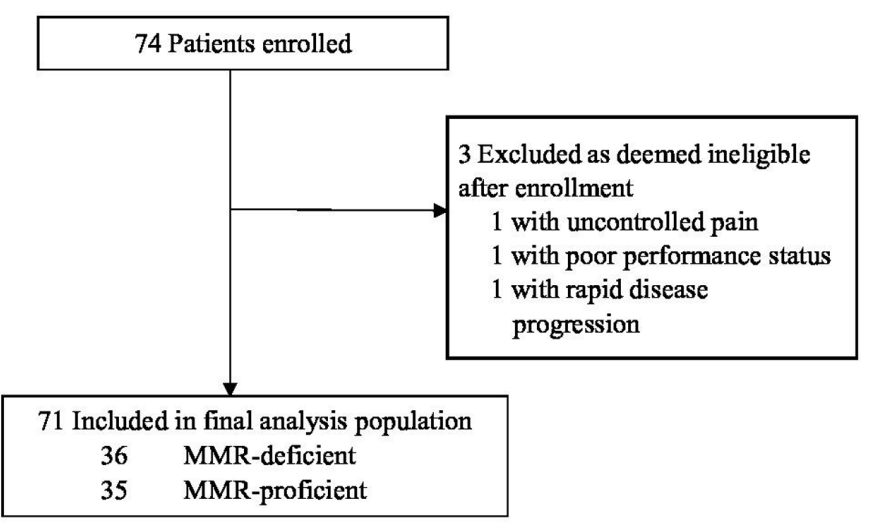

Figure 1 Enrollment and outcomes. MMR, mismatch mutation repair.

\begin{tabular}{|c|c|c|}
\hline Characteristic & $\begin{array}{l}\text { dMMR } \\
(n=36)\end{array}$ & $\begin{array}{l}\text { pMMR } \\
(n=35)\end{array}$ \\
\hline Median age (years) (range) & $66(36-76)$ & $68(37-81)$ \\
\hline \multicolumn{3}{|l|}{ ECOG, n (\%) } \\
\hline 0 & $18(50)$ & $17(49)$ \\
\hline 1 & $15(42)$ & $18(51)$ \\
\hline 2 & $3(8)$ & 0 \\
\hline \multicolumn{3}{|l|}{ Grade at diagnosis, $\mathrm{n}(\%)^{\star}$} \\
\hline 1 & $9(26)$ & $6(17)$ \\
\hline 2 & $17(49)$ & $3(9)$ \\
\hline 3 & $9(26)$ & $26(74)$ \\
\hline \multicolumn{3}{|l|}{ Pathology, n (\%) } \\
\hline Endometrioid & $34(94)$ & $20(57)$ \\
\hline Serous & 0 & $11(31)$ \\
\hline Clear cell & $1(3)$ & $1(3)$ \\
\hline Others & 0 & $3(9)$ \\
\hline \multicolumn{3}{|l|}{ Hormone status, n (\%) } \\
\hline ER positive† & $26 / 28(93)$ & $19 / 29(66)$ \\
\hline PR positive† & $21 / 25(84)$ & $14 / 24(58)$ \\
\hline Prior surgery, n (\%) & $32(89)$ & $31(89)$ \\
\hline Prior radiotherapy, n (\%) & $27(75)$ & $20(57)$ \\
\hline \multicolumn{3}{|c|}{ Lines of prior systemic treatment for advanced disease, $n(\%) \ddagger$} \\
\hline 0 & $21(60)$ & $3(8)$ \\
\hline 1 & $14(39)$ & $22(63)$ \\
\hline$\geq 2$ & $1(3)$ & $10(27)$ \\
\hline \multicolumn{3}{|l|}{ Prior chemotherapy, n (\%) } \\
\hline Platinum doublet & $19(53)$ & $33(94)$ \\
\hline Platinum monotherapy & $3(9)$ & $5(14)$ \\
\hline Taxane monotherapy & 0 & $3(8)$ \\
\hline Doxorubicin/liposomal doxorubicin & $1(3)$ & $4(11)$ \\
\hline Other chemotherapy & $1(3)$ & $2(6)$ \\
\hline Previous hormonal therapy, n (\%) & $2(6)$ & $5(14)$ \\
\hline Previous bevacizumab, n (\%) & 0 & $2(6)$ \\
\hline
\end{tabular}

*One missing value.

†Denominators given due to missing values.

$\ddagger$ Excluding hormone, bevacizumab, adjuvant and neoadjuvant chemotherapy received $\geq 12$ months prior to registration.

dMMR, mismatch repair deficient; ECOG, Eastern Cooperative Oncology Group; ER, Estrogen Receptor ; pMMR, mismatch repair proficient; PR, Progesterone receptor.

reported in $56 \%$ of the dMMR cohort, while most of the pMMR cohort $(97 \%)$ had prior platinum-based doublet or single-agent systemic therapy. Four women enrolled in the pMMR cohort were later found to have protocol violation (three had no prior chemotherapy for advanced disease prior to receiving durvalumab and one had carcinosarcoma) but were included in all analyses as they were deemed eligible at the time of enrollment and underwent treatment. 
Table 2 Objective tumor response by iRECIST

\begin{tabular}{|c|c|c|c|c|}
\hline & dMMR $(n=36)$ & & pMMR & \\
\hline Best OTRR & 17 & $47 \%$ (95\% $\mathrm{Cl} 32 \%$ to $63 \%)$ & 1 & $3 \%(95 \% \mathrm{Cl} 1 \%$ to $15 \%)$ \\
\hline \multicolumn{5}{|c|}{ OTRR by lines of prior chemotherapy, $n$ (\%) } \\
\hline 0 & $12(57)$ & & $0(0)$ & \\
\hline 1 & $5(38)$ & & $1(5)$ & \\
\hline $2 />$ & $0(0)$ & & $0(0)$ & \\
\hline \multicolumn{5}{|c|}{ Best overall response, $\mathrm{n}(\%)$} \\
\hline iCR & $6(17)$ & & $0(0)$ & \\
\hline iPR & $11(31)$ & & $1(3)$ & \\
\hline iSD & $6(17)$ & & $10(29)$ & \\
\hline iCPD & $13(36)$ & & $23(66)$ & \\
\hline DCR at 16 weeks & $21(58)$ & & $8(23)$ & \\
\hline DCR at 24 weeks & $18(50)$ & & $7(20)$ & \\
\hline
\end{tabular}

$\mathrm{DCR}$, disease control rate; dMMR, mismatch repair deficient; iCPD, confirmed progressive disease by iRECIST; iCR, complete response by iRECIST; iPR, partial response by iRECIST; iRECIST, RECIST V.1.1 modified for immune-based therapeutics; iSD, stable disease by iRECIST; OTRR, objective tumor response rate; pMMR, mismatch repair proficient.

\section{Efficacy analysis}

As of the data cut-off on August 10, 2019, 15 of 71 women (14 dMMR and 1 pMMR) were still on durvalumab. Seventy women were evaluable for response (35 in each cohort). The remaining woman died of rapid tumor progression before starting treatment with durvalumab. The median follow-up times were 19 months for the dMMR cohort and 21 months for the pMMR cohort.

Tumor response data are summarized in table 2. In the dMMR cohort, the OTRR (iRECIST) was $47 \%$ (95\% CI $32 \%$ to $63 \% ; 17 / 36$, including 6 CR and 11 PR; figure 2 ). In the pMMR cohort, the OTRR was $3 \%(95 \%$ CI $1 \%$ to $15 \%, 1 / 35$ with PR). In the dMMR cohort, OTRR was $57 \%$ in those not previously treated with chemotherapy vs $38 \%$ in those previously treated with chemotherapy. Disease control rates (according to iRECIST) at 16 and at 24 weeks, respectively, were $58 \%$ and $50 \%$ in the dMMR cohort vs $23 \%$ and $20 \%$ in the pMMR cohort. The results were similar with assessments using RECIST (online supplemental table $\mathrm{S} 1$ ).

The 6-month PFS rate based on iRECIST was 53\% (95\% CI $36 \%$ to $67 \%$ ) in the dMMR cohort and $14 \%$ (95\% CI $5 \%$ to $28 \%$ ) in the pMMR cohort (figure 3 ). Median PFS based on iRECIST was 8.3 months (95\% CI 2.4 to NR) in the dMMR cohort and 1.8 months (95\% CI 1.8 to 2.0 ) in the pMMR cohort. At the data cut-off, only 2 of the 18 responders in the dMMR cohort had progressed. Duration of response in the other 16 women ranged from 8 to 20 months.

The 12-month OS rates were $71 \%$ in the dMMR cohort and $51 \%$ in the pMMR cohort (figure 3), with median OS not reached for dMMR vs 12.1 months for pMMR. Results of PFS based on RECIST V.1.1 are presented in online supplemental figure S1.

\section{Safety}

Sixty-nine women received at least one dose of durvalumab. Overall, $93 \%$ women experienced at least
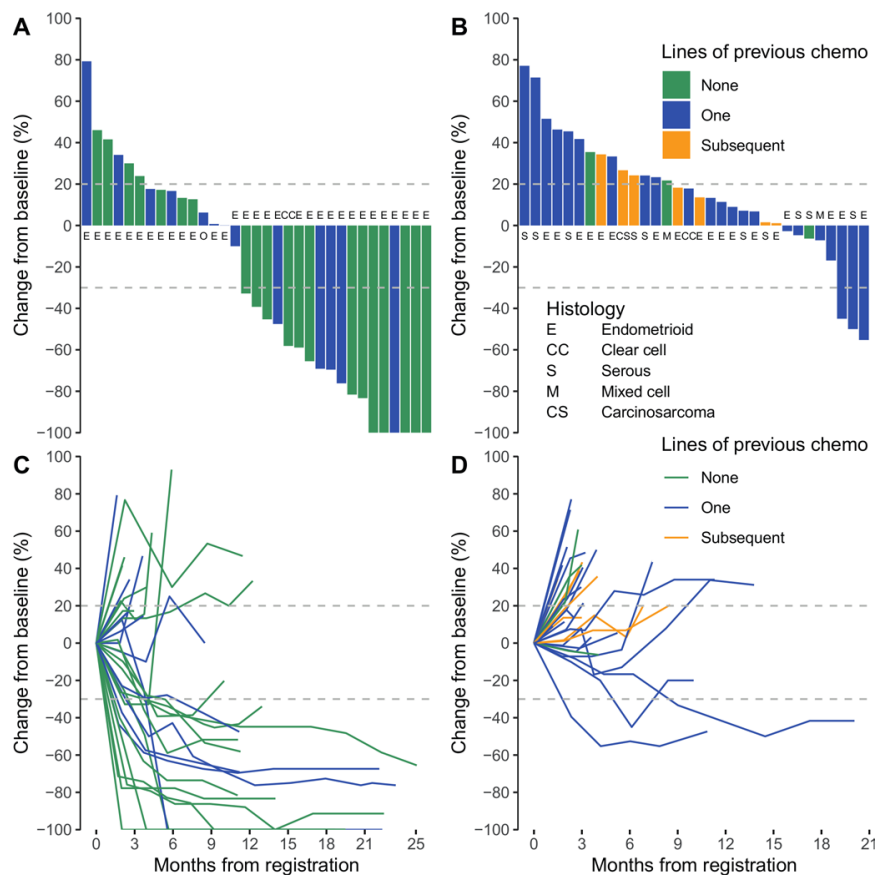

Figure 2 Objective tumor responses in dMMR and pMMR advanced endometrial cancer cohorts. Waterfall plots for the maximum decrease in the sum of the target lesions are given in (A) for dMMR ( $n=32,2$ died prior to reassessment and 1 was not evaluable for target lesions) and (B) for pMMR ( $n=33$, 3 died prior to reassessment), colored by lines of previous chemotherapy, with histology marked. Spider plots for the change in the sum of the target lesions over time are shown for dMMR $(n=32)(C)$ and for pMMR $(n=33)(D)$, colored by lines of previous chemotherapy. dMMR, mismatch repair deficient; pMMR, mismatch repair proficient. 
A

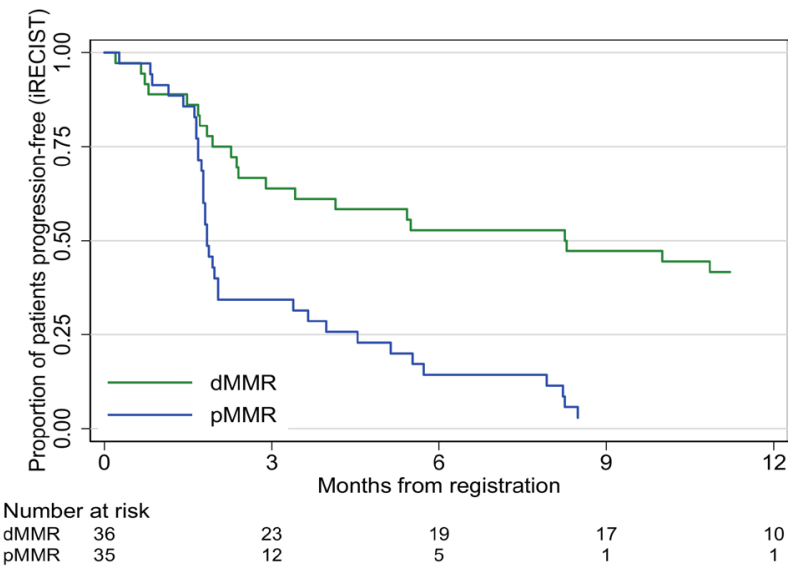

B

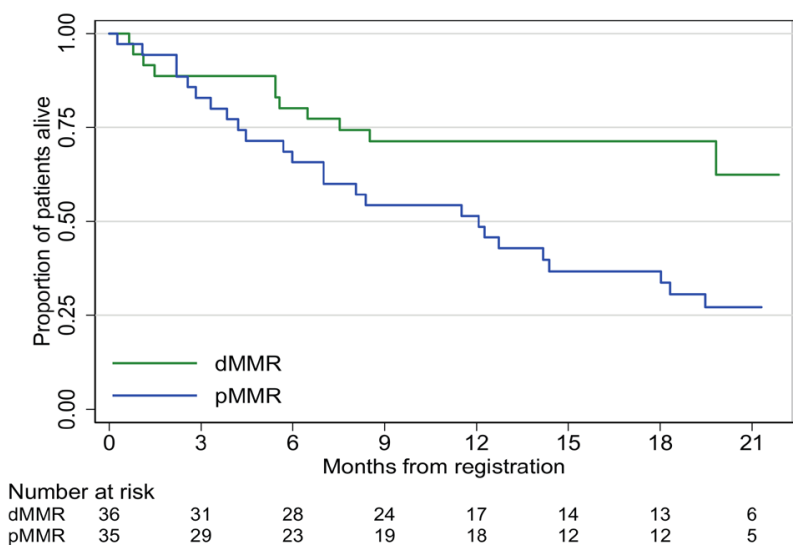

Figure 3 Graphs of (A) PFS (B) OS in dMMR and pMMR cohorts. dMMR, mismatch repair deficient; iRECIST, RECIST V.1.1 modified for immune-based therapeutics; pMMR, mismatch repair proficient. OS, overall survival; PFS, progression-free survival.

one AE (all grades). There were 19 immune-related adverse events (irAEs) reported in 14 women (20\%), 7 in each cohort (table 3 ). These were mainly of grades 1 and $2(n=18)$, including hypothyroidism $(n=7)$, hyperthyroidism $(n=8)$, pneumonitis $(n=2)$ and hypoadrenalism $(n=1)$. Only one woman experienced a grade 3 irAE (hepatitis). All other reported AEs were judged unrelated to durvalumab by investigators (online supplemental table S2). Three women ceased treatment in relation to an $\mathrm{AE}$, one of which was an associated irAE, the other two relating to the development of a second primary tumor and a brain abscess.

\section{Quality of life}

Completion rates for HRQL questionnaires were high, with $92 \%$ of expected assessments completed at baseline and at 3 months (online supplemental table S3). An improvement of 10 points or greater from baseline maintained for two or more visits was observed for global health and quality of life domain in $9 / 36(25 \%)$ in the dMMR cohort and 3/35 (9\%) in the pMMR cohort (online supplemental table S4). Similar improvements in pain were observed in 12/36 (33\%) in the dMMR cohort and 3/35 (10\%) in the pMMR cohort. The quality of life status at 3 and 6 months by MMR status is shown in online supplemental table S5. The mean scores over time for pain, fatigue, nausea and vomiting, dsypnea and diarrhea are reported in online supplemental figures S2S6. Scores for fatigue and dyspnea appeared better in the dMMR cohort compared with the pMMR cohort.

\section{DISCUSSION}

The PHAEDRA trial showed that treatment with the single-agent anti-PD-L1 antibody, durvalumab, was active in women with dMMR AEC, who were either chemotherapy naive or had at least one previous line of chemotherapy, with an OTRR of $47 \%$. Responses were often durable and six CRs were seen. There was minimal activity in the pMMR cohort, with an OTRR of 3\%. Durvalumab was well tolerated, with most irAEs being of grade 1 or 2 , with only one woman discontinuing treatment due to a treatment-related irAE.

These results are consistent with trials of other singleagent immune checkpoint inhibitors (mainly PD-1

Table 3 Immune-related adverse events by MMR cohort

\begin{tabular}{|c|c|c|c|c|}
\hline & \multicolumn{2}{|l|}{ dMMR } & \multicolumn{2}{|l|}{ pMMR } \\
\hline & Any grade & Grade 3 or higher & Any grade & Grade 3 or higher \\
\hline $\begin{array}{l}\text { Eligible patients who received at least } \\
\text { one dose of study treatment }\end{array}$ & 35 & & 34 & \\
\hline $\begin{array}{l}\text { Patients who experienced at least one } \\
\text { immune-related event, } n(\%)\end{array}$ & $7(20)$ & & $7(21)$ & $1(3)$ \\
\hline \multicolumn{5}{|l|}{ Immune-related events, $\mathrm{n}(\%)$} \\
\hline Hyperthyroidism & $5(14)$ & & $2(6)$ & \\
\hline Hypothyroidism & $3(9)$ & & $3(9)$ & \\
\hline Pneumonitis & $1(3)$ & & $1(3)$ & \\
\hline Adrenal insufficiency & $1(3)$ & & 0 & \\
\hline Viral hepatitis & 0 & & $1(3)$ & $1(3)$ \\
\hline
\end{tabular}

dMMR, mismatch repair deficient; MMR, mismatch mutation repair; pMMR, mismatch repair proficient. 
inhibitors) in AEC. ${ }^{21} 23$ 26-30 Avelumab, assessed in a similar two-cohort study of dMMR and pMMR AECs, was reported to have OTRRs of $27 \%$ and $6 \%$, respectively, with the pMMR cohort closed early for futility. ${ }^{27}$ Pembrolizumab has been reported in two phase II studies. Le et al reported an OTRR of 53\% (46/86) in women with dMMR tumors, including $18(21 \%)$ with CR. ${ }^{26}$ KEYNOTE-158 reported an OTRR of 57\%, including eight (18\%) CRs. ${ }^{28}$ A study of another a PD-1 inhibitor, dostarlimab (TSR042), in AEC reported an OTRR of $49 \%$ in MSI-H and $20 \%$ in Microsatelite Stable (MSS). ${ }^{29} 30$

It is important to note that these earlier studies ${ }^{21} 23$ selected MSI subjects according to sequencing, whereas eligibility for the later studies were based on either IHC assessment for MMR expression or sequencing to assess microsatellite stability. ${ }^{27}$ The dichotomy of OTR according to MMR/MSI status emphasizes the importance of such assessments. Microsatellite testing generally requires both normal and tumor tissues and access to a sequencing platform, and is more time-consuming. In contrast, IHC assessment for MMR is more widely available and less expensive. Pathology review revised the site-defined MMR status in only one subject with confirmed loss of MLH1 and PMS2, indicating a high level of appropriate interpretation of MMR expression. National Comprehensive Cancer Network (NCCN) guidelines recommend universal IHC assessment for MMR in all women with AEC. ${ }^{31}$ Up to $7 \%$ cases were found to have discordance between the metastatic tumor and the matched primary cancer. ${ }^{32}$ Potential discordance and treatment implications support repeat testing when feasible.

Tumors that demonstrate dMMR often have an increased mutational load and are largely, but not always, associated with MSI. ${ }^{33}$ As has been reported for other dMMR/MSI-H tumor types, ECs demonstrating either dMMR or MSI-H tumors are associated with higher response rates to inhibition of PD-1 or PD-L1. One of the hallmarks of dMMR/MSI-H tumors is lymphocytic infiltration, with more recent studies demonstrating enriched expression of PD-1, PD-L1, CTLA-4, LAG-3 and IDO, suggestive of highly 'primed' tumors. Blockade of PD-1/ PD-L1 in these highly primed tumors may increase the cytotoxic immune response by inducing Th-1-mediated cancer cell destruction. ${ }^{27}{ }^{34}$ Le et al hypothesized that the primary reason for higher response rates to PD-1 inhibition in MSI-H tumors is the higher frequency of mutationassociated neoantigens resulting from MSI (20 times that of MSS tumors). ${ }^{21}$ The results of this study support the notion that mismatch repair deficiency is an important predictor of increased response to checkpoint inhibition. However, it remains to be established why some dMMR tumors are resistant to PD-1 or PD-L1 inhibition.

In many tumor types, the response to treatment with a single-agent, immune checkpoint inhibitor diminishes with an increasing number of previous lines of treatment. In PHAEDRA, the OTRR with durvalumab was higher as first-line treatment than as second or subsequent line (57\% vs $38 \%$ ). Indeed, the timing of PD-L1 inhibition may have also affected the likelihood of response in the pMMR cohort, where all subjects had progressed after treatment with chemotherapy. Many factors associated with more advanced disease, including reduced performance status, reduced host immune responsiveness altered tumor microenvironment, and exposure to prior therapies, might reduce the effects of PD-L1 inhibition. Chemotherapy might induce immune vulnerability by causing apoptosis and increasing tumor antigen presentation, particularly when soon before treatment with inhibitors of PD-1/PD-L1. However, over an extended period, treatment with chemotherapy and subsequent resistance to cytotoxics might be associated with reduced beneficial effects of single-agent PD-L1 inhibition. ${ }^{35}$

Immunotherapy appears to be a promising option for dMMR AEC but not pMMR AEC. An exception may be pMMR AEC with a POLE-hypermutated phenotype, which may be very sensitive to immune checkpoint inhibition. ${ }^{36}$ Inducing an immune response to checkpoint inhibitors by combining them with other treatments may be a more rational approach for pMMR tumors. A trial of combining the multikinase inhibitor lenvatinib plus pembrolizumab reported an OTRR of $40 \%$ (95\% CI $26.5 \%$ to $54.0 \%$ ) at week $24 .{ }^{37}$ Most subjects had pMMR tumors, and responses were seen in those with and without tumor expression of PD-1. There are no additional data available in terms of patient characteristics that differentiate the modest responses seen in pMMR tumors in the PHAEDRA and other PD-1/PD-L1 inhibitor studies compared with the combined lenvatinib-pembrolizumab combination. ${ }^{37}$ Toxicity was significant with grade 3 or 4 treatment-related AEs seen in $67 \%$ of subjects. In preclinical models, the combination was associated with alterations in the tumor-associated immune infiltrates that were associated with improved antitumor activity of PD-1 inhibition. ${ }^{38}$ Additional strategies that may prime endometrial tumors for immune susceptibility might include treatment with cytotoxic drugs, radiotherapy, other antiangiogenic drugs, PARP inhibitors, and/or AKT inhibitors. $^{35}$

We observed an OTRR in dMMR tumors similar to that reported with first-line chemotherapy using carboplatin and paclitaxel, the current standard of care, and demonstrated the importance of assessing MMR status when considering treatment options for AEC. Ongoing clinical trials that are exploring the addition of PD-1/PD-L1 inhibitors to standard chemotherapy will help clarify the potential for chemotherapy benefits of adding immunotherapy in both pMMR and dMMR AECs. These studies are based on chemotherapy as the standard control treatment, testing the addition of PD-1 or PD-L1 inhibition as the investigational arm. Whether or not PD-L1 inhibition alone might be as effective as, and less toxic than, chemotherapy in dMMR AEC will not be answered by these trials. Further research is needed to determine how to increase response rates in this immune-susceptible tumor type, given that responses to single-agent PD-1/PD-L1 inhibitors are not seen in all dMMR ECs. Combining 
checkpoint inhibitors with other immune-enhancing agents may prove beneficial.

The main limitation of this study include the small number of women in each cohort and the lack of a randomly allocated control group. Additionally, while typical of this molecular subtype, most tumors in the dMMR cohort were of endometrioid histology. Of the non-endometrioid histologies in this cohort, one woman with a clear cell carcinoma achieved a PR, and one woman with a serous carcinoma showed no response to durvalumab. Further exploration of these rarer subtypes and their susceptibility to PD-L1 inhibition is required. Research to identify other biomarkers associated with response is ongoing.

In conclusion, PHAEDRA showed that durvalumab has encouraging activity and tolerability in dMMR AEC but minimal activity in pMMR AEC. This warrants further clinical trials comparing durvalumab with chemotherapy and or other immune-modulating agents in women with dMMR EC and further research seeking to induce immune responses in pMMR tumors.

\section{Author affiliations}

${ }^{1}$ Medical Oncology, Cabrini Health, Malvern, Victoria, Australia

${ }^{2}$ Faculty of Medicine, Dentistry and Health Sciences, Monash University, Clayton, VIC, Australia

${ }^{3}$ NHMRC Clinical Trials Centre, University of Sydney, Camperdown, New South Wales, Australia

${ }^{4}$ Mater Pathology, Mater Research and University of Queensland, Brisbane, Queensland, Australia

${ }^{5}$ Molecular Cancer Epidemiology Laboratory, QIMR Berghofer Medical Research Institute, Herston, Queensland, Australia

${ }^{6}$ Department of Medical Oncology, Prince of Wales Hospital Nelune Comprehensive Cancer Centre, Randwick, New South Wales, Australia

${ }^{7}$ Chris 0'Brien Lifehouse, Camperdown, New South Wales, Australia

${ }^{8}$ Medical Oncology, Royal North Shore Hospital, St Leonards, New South Wales,

Australia

${ }^{9}$ Mater Cancer Care Centre, Mater Hospital, South Brisbane, Queensland, Australia

${ }^{10}$ Clinical Trials Unit, Icon Cancer Care, South Brisbane, Queensland, Australia

${ }^{11}$ School of Medicine, University of Queensland, St Lucia, QLD, Australia

${ }^{12}$ Medical Oncology, Monash Medical Centre Clayton, Clayton, Victoria, Australia

${ }^{13}$ Department of Medical Oncology, Sir Charles Gairdner Hospital, Nedlands,

Western Australia, Australia

${ }^{14}$ Medical Oncology, Calvary Mater Newcastle, Hunter Region Mail Centre, New

South Wales, Australia

${ }^{15}$ Department of Medical Oncology, Peter MacCallum Cancer Centre, Parkville, Victoria, Australia

Acknowledgements We thank the subjects, their families and caregivers, the investigators, and staff at participating sites, the NHMRC Clinical Trials Centre and the Australia New Zealand Gynecological Oncology Group.

Collaborators Australia New Zealand Gynaecological Oncology Group (ANZGOG)

Contributors YA: conceptualization, resources, supervision, visualization, investigation, and writing (original draft); P-SK: conceptualization, data curation, formal analysis, investigation, validation, methodology, project administration, software and writing (review and editing); KR: formal analysis, investigation, methodology, validation and writing (review and editing); MC: project administration and data curation; DS: formal analysis, investigation and writing (review and editing); AS: investigation and writing (review and editing); EB: formal analysis, investigation and methodology; YCL: writing (review and editing); MF: conceptualization, supervision and writing (review and editing); SY, SB-H, CS, JC, PB, GG, TM, JL and JA: data curation and investigation; MS: conceptualization, methodology, supervision and writing (review and editing); LM: conceptualization, supervision, resources and writing (review and editing).
Funding This investigator-initiated, academically led cooperative group trial was funded by AstraZeneca. AS was supported by a National Health and Medical Research Council (Australia) Fellowship funding (ID1061778 and ID1177524). AS holds a National Health and Medical Research Council of Australia Fellowship grant

Competing interests YA: honoraria from AstraZeneca and research funding from AstraZeneca. P-SK: research funding from AstraZeneca and honoraria from Pfizer. DS: stock or other ownership for SNP Pathology; honoraria from Mark Sharp \& Dohme; and patents, royalties, and other intellectual property from Uniquest. MF: honoraria from Pfizer and AstraZeneca; and consulting or advisory role, or AstraZeneca and Pfizer. SB-H: consulting or advisory role from AstraZeneca, Novartis, and Pfizer; and travel and accommodation expenses from Novartis. CS: speakers' bureau for AstraZeneca olaparib advisory board, Roche HER2+ breast cancer. JC: honoraria from Takeda, MSD; consulting or advisory role from Takeda, MSD; research funding from AstraZeneca; and travel, accommodation, and expenses from AMGEN-ESM0. PB: honoraria from Roche; consulting or advisory role from AstraZeneca; and travel and accommodation expenses from Astra Zeneca. TM: honoraria and research funding from AstraZeneca. MRS: research funding from Astra Zeneca.

Patient consent for publication Not required.

Ethics approval The protocol was approved by a central human research ethics committee (HREC) and all site HRECs.

Provenance and peer review Not commissioned; externally peer reviewed.

Data availability statement Data are available upon reasonable request. All data relevant to the study are included in the article or uploaded as supplemental information. Individual deidentified subject data that underlie the results reported in this article will be shared upon reasonable request. Study protocol has been shared. Additional related documents will be available (eg, s statistical analysis plan) upon reasonable request.

Supplemental material This content has been supplied by the author(s). It has not been vetted by BMJ Publishing Group Limited (BMJ) and may not have been peer-reviewed. Any opinions or recommendations discussed are solely those of the author(s) and are not endorsed by BMJ. BMJ disclaims all liability and responsibility arising from any reliance placed on the content. Where the content includes any translated material, BMJ does not warrant the accuracy and reliability of the translations (including but not limited to local regulations, clinical guidelines, terminology, drug names and drug dosages), and is not responsible for any error and/or omissions arising from translation and adaptation or otherwise.

Open access This is an open access article distributed in accordance with the Creative Commons Attribution Non Commercial (CC BY-NC 4.0) license, which permits others to distribute, remix, adapt, build upon this work non-commercially, and license their derivative works on different terms, provided the original work is properly cited, appropriate credit is given, any changes made indicated, and the use is non-commercial. See http://creativecommons.org/licenses/by-nc/4.0/.

\section{ORCID iD}

Yoland Antill http://orcid.org/0000-0002-6366-5523

\section{REFERENCES}

1 Bokhman JV. Two pathogenetic types of endometrial carcinoma. Gynecol Oncol 1983;15:10-17.

2 Cancer Genome Atlas Research Network, Kandoth C, Schultz N, et al. Integrated genomic characterization of endometrial carcinoma. Nature 2013;497:67-73.

3 Kandoth C, McLellan MD, Vandin F, et al. Mutational landscape and significance across 12 major cancer types. Nature 2013;502:333-9.

4 Nagle CM, O'Mara TA, Tan Y, et al. Endometrial cancer risk and survival by tumor MMR status. J Gynecol Oncol 2018;29:e39.

5 Buchanan DD, Rosty C, Clendenning M, et al. Clinical problems of colorectal cancer and endometrial cancer cases with unknown cause of tumor mismatch repair deficiency (suspected Lynch syndrome). Appl Clin Genet 2014;7:183-93.

6 Buchanan DD, Tan YY, Walsh MD, et al. Tumor mismatch repair immunohistochemistry and DNA MLH1 methylation testing of patients with endometrial cancer diagnosed at age younger than 60 years optimizes triage for population-level germline mismatch repair gene mutation testing. J Clin Oncol 2014;32:90-100.

7 Baglietto L, Lindor NM, Dowty JG, et al. Risks of Lynch syndrome cancers for MSH6 mutation carriers. J Natl Cancer Inst 2010;102:193-201. 
8 Senter L, Clendenning M, Sotamaa K, et al. The clinical phenotype of Lynch syndrome due to germ-line PMS2 mutations. Gastroenterology 2008;135:419-28. e1.

9 Dowty JG, Win AK, Buchanan DD, et al. Cancer risks for MLH1 and MSH2 mutation carriers. Hum Mutat 2013;34:490-7.

10 Kempers MJE, Kuiper RP, Ockeloen CW, et al. Risk of colorectal and endometrial cancers in EPCAM deletion-positive Lynch syndrome: a cohort study. Lancet Oncol 2011;12:49-55.

11 Roudko V, Bozkus CC, Orfanelli T, et al. Shared immunogenic PolyEpitope frameshift mutations in microsatellite unstable tumors. Cell 2020;183:1634-49. e17.

12 León-Castillo A, de Boer SM, Powell ME, et al. Molecular classification of the PORTEC-3 trial for high-risk endometrial cancer: impact on prognosis and benefit from adjuvant therapy. $J$ Clin Oncol 2020;38:3388-97.

13 Lee V, Murphy A, Le DT, et al. Mismatch repair deficiency and response to immune checkpoint blockade. Oncologist 2016;21:1200-11.

14 Dolcetti R, Viel A, Doglioni C, et al. High prevalence of activated intraepithelial cytotoxic T lymphocytes and increased neoplastic cel apoptosis in colorectal carcinomas with microsatellite instability. Am J Pathol 1999;154:1805-13.

15 Pasanen A, Ahvenainen T, Pellinen T, et al. PD-L1 expression in endometrial carcinoma cells and intratumoral immune cells: differences across histologic and TCGA-based molecular subgroups. Am J Surg Pathol 2020;44:174-81.

16 Topalian SL, Drake CG, Pardoll DM. Targeting the PD-1/B7H1(PD-L1) pathway to activate anti-tumor immunity. Curr Opin Immunol 2012;24:207-12.

17 Sutton GP, Blessing JA, DeMars LR, et al. A phase II gynecologic Oncology Group trial of ifosfamide and mesna in advanced or recurrent adenocarcinoma of the endometrium. Gynecol Oncol 1996;63:25-7.

18 Wadler S, Levy DE, Lincoln ST, et al. Topotecan is an active agent in the first-line treatment of metastatic or recurrent endometrial carcinoma: eastern cooperative Oncology Group study E3E93. J Clin Oncol 2003;21:2110-4.

19 Muggia FM, Blessing JA, Sorosky J, et al. Phase II trial of the pegylated liposomal doxorubicin in previously treated metastatic endometrial cancer: a gynecologic Oncology Group study. J Clin Oncol 2002;20:2360-4.

20 Fracasso PM, Blessing JA, Molpus KL, et al. Phase II study of oxaliplatin as second-line chemotherapy in endometrial carcinoma: a gynecologic Oncology Group study. Gynecol Oncol 2006;103:523-6.

21 Le DT, Uram JN, Wang H, et al. PD-1 blockade in tumors with mismatch-repair deficiency. N Engl J Med 2015;372:2509-20.

22 U.S. Food and Drug Administration. FDA grants accelerated approval to pembrolizumab for first tissue/site agnostic indication.

23 Ott PA, Bang Y-J, Berton-Rigaud D, et al. Safety and antitumor activity of pembrolizumab in advanced programmed death ligand 1-positive endometrial cancer: results from the KEYNOTE-028 study. J Clin Oncol 2017;35:2535-41.
24 Eisenhauer EA, Therasse P, Bogaerts J, et al. New response evaluation criteria in solid tumours: revised RECIST guideline (version 1.1). Eur J Cancer 2009;45:228-47.

25 Seymour L, Bogaerts J, Perrone A, et al. iRECIST: quidelines for response criteria for use in trials testing immunotherapeutics. Lancet Oncol 2017;18:e143-52.

26 Le DT, Durham JN, Smith KN, et al. Mismatch repair deficiency predicts response of solid tumors to PD-1 blockade. Science 2017;357:409-13.

27 Konstantinopoulos PA, Luo W, Liu JF, et al. Phase II study of Avelumab in patients with mismatch repair deficient and mismatch repair proficient Recurrent/Persistent endometrial cancer. J Clin Oncol 2019;37:2786-94.

28 Marabelle A, Le DT, Ascierto PA, et al. Efficacy of pembrolizumab in patients with Noncolorectal high microsatellite Instability/Mismatch repair-deficient cancer: results from the phase II KEYNOTE-158 study. J Clin Oncol 2020;38:1-10.

29 Oaknin A, Tinker AV, Gilbert L, et al. Clinical activity and safety of the Anti-Programmed death 1 monoclonal antibody Dostarlimab for patients with recurrent or advanced mismatch repair-deficient endometrial cancer: a nonrandomized phase 1 clinical trial. JAMA Oncol 2020;6:1766-72.

30 Oaknin A, Ellard SL, Leath III C, et al. Preliminary safety, efficacy, and PK/PD characterization from GARNET, a phase I clinical trial of the anti-PD-1 monoclonal antibody, TSR-042, in patients with recurrent or advanced $\mathrm{MSI}-\mathrm{H}$ endometrial cancer. Ann Oncol 2018;29:viii334.

31 Koh W-J, Abu-Rustum NR, Bean S, et al. Uterine neoplasms, version 1.2018, NCCN clinical practice guidelines in oncology. J Nat/ Compr Canc Netw 2018;16:170-99.

32 Ta RM, Hecht JL, Lin DI. Discordant loss of mismatch repair proteins in advanced endometrial endometrioid carcinoma compared to paired primary uterine tumors. Gynecol Oncol 2018;151:401-6.

33 Cancer Genome Atlas Research Network, Weinstein JN, Collisson $\mathrm{EA}$, et al. The cancer genome atlas pan-cancer analysis project. Nat Genet 2013;45:1113-20.

34 Topalian SL, Drake CG, Pardoll DM. Immune checkpoint blockade: a common denominator approach to cancer therapy. Cancer Cell 2015;27:450-61.

$35 \mathrm{Li}$ X, Shao C, Shi Y, et al. Lessons learned from the blockade of immune checkpoints in cancer immunotherapy. $J$ Hematol Oncol 2018;11:31.

36 Mehnert JM, Panda A, Zhong H, et al. Immune activation and response to pembrolizumab in POLE-mutant endometrial cancer. $J$ Clin Invest 2016;126:2334-40.

37 Makker V, Rasco D, Vogelzang NJ, et al. Lenvatinib plus pembrolizumab in patients with advanced endometrial cancer: an interim analysis of a multicentre, open-label, single-arm, phase 2 trial. Lancet Oncol 2019;20:711-8.

38 Kato Y, Bao X, Macgrath S, et al. Lenvatinib mesilate (LEN) enhanced antitumor activity of a PD-1 blockade agent by potentiating Th1 immune response. Ann Oncol 2016;27:vi1. 